\title{
A Study on Vibration Characteristics and Stability of the Ambulance Nonlinear Damping System
}

\author{
Meng Yang, Xinxi Xu, and Chen Su \\ Institute of Medical Equipment, Academy of Military Medical Sciences, 106 Wandong Road, Hedong District, Tianjin 300161, China \\ Correspondence should be addressed to Xinxi Xu; xuxxl@sohu.com
}

Received 28 December 2012; Revised 25 March 2013; Accepted 22 April 2013

Academic Editor: Chunrui Zhang

Copyright (C) 2013 Meng Yang et al. This is an open access article distributed under the Creative Commons Attribution License, which permits unrestricted use, distribution, and reproduction in any medium, provided the original work is properly cited.

\begin{abstract}
Considering the impact of the nonlinear stiffness, a 2-DOF vibration model with cubic terms was established according to the structural feature and nonlinear behavior. Ignoring the impact of nonlinear terms, the system was linearly analyzed. In the case of primary resonance and $1: 1$ internal resonance, a multiscale method was used to obtain a first-order approximate solution. Taking the parameters of one tracked ambulance for instance, the approximate solution was corroborated and the influence of the parameters on damping effect was investigated. Finally, motion stability of the damping system was analyzed with singularity theory. The theoretical bases for improving efficiency of the damping system were provided.
\end{abstract}

\section{Introduction}

The tracked ambulance can go through a variety of complex terrain and perform first aid on the sick and wounded. To achieve safe transfer and implement first aid on the way, it is often necessary to demand good mobility of tracked ambulance and meet the special needs of the sick and wounded. For the tracked ambulance that is refitted by crawler chassis, the installation of the vehicle damping system becomes the main way to improve the ride comfort of the sick and wounded.

The tracked ambulance damping system is comprised of the carriage, the stretcher base, the chassis, and the nonlinear shock absorber. Therefore, it can be easily converted into a multidegree-of-freedom nonlinear vibration system. The use of the nonlinear vibration system presents numerous advantages, such as better performance in inhibiting broadband vibration, especially low-frequency vibration. However, complex mechanical properties usually exist in a nonlinear vibration system such as chaos and bifurcation, which makes it difficult to be analytically calculated and analyzed; therefore approximate analytical algorithm are widely used. Lee and Perkins [1] investigatesuspended, elastic cables driven by planar excitation with nearly commensurable natural frequencies in a 2:1 ratio. The first-order analysis shows that there are saturation and jump phenomena, and the cubic nonlinearity disrupts saturation. Li et al. [2] apply multiple scales method and Runge-Kutta to study the nonlinear vibration characteristics of the axial movement, multilayered cylindrical shells made from composites. The results show some nonlinear properties of the system, such as the phenomenon of internal resonance, and point out that the excitation amplitude, damping, and velocity can affect the response amplitude, range of internal resonance, and soft feature of the system. Xin et al. [3] use the average method to analyze piecewise nonlinear characteristics of the viscoelastic shocker absorber and the relationship between amplitude-frequency characteristics and system parameters. Xinye et al. [4] use the average method to study the possibility of delay feedback control over the gyroscope system under force. Inoue and Ishida [5] investigate vibration phenomena of the one-degree-offreedom magnetically levitated system considering the effect of the nonlinearity of the electromagnet, using a shooting method. Moon and Kang [6] put forward an analytical method for evaluation of the steady-state periodic behavior of a nonlinear system. The method is based on the substructure synthesis formulation and a multiple-scale procedure.

In this paper, the differential equations of the 2-DOF tracked ambulance nonlinear damping system, including the cubic nonlinear spring, were presented. In the case of primary 


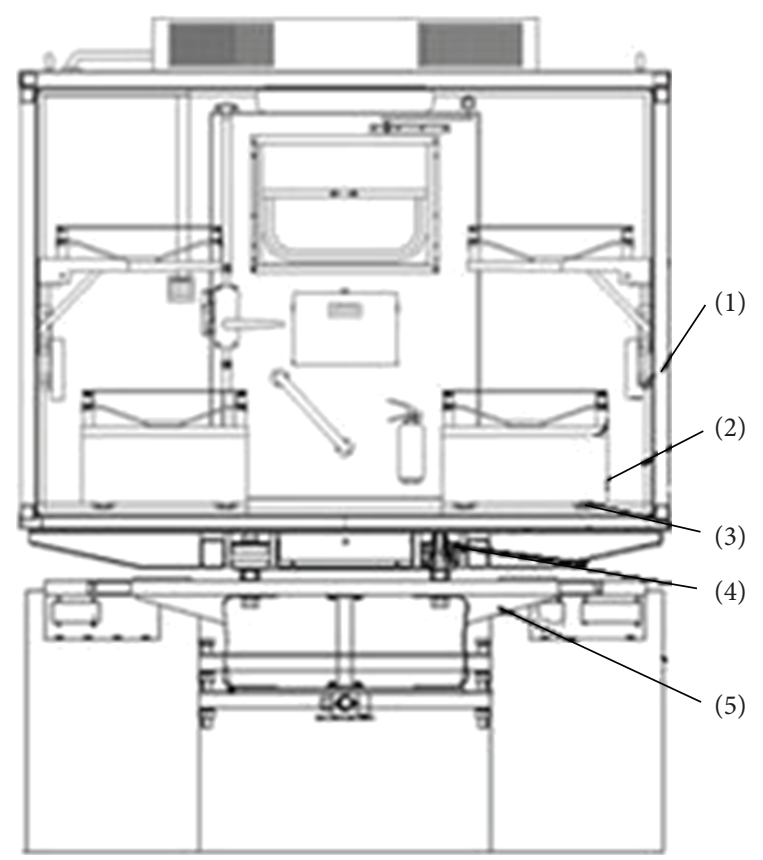

Figure 1: Ambulance damping system: (1) carriage, (2) stretcher base, (3) zero stiffness damper, (4) rubber damping shock absorber, and (5) coach chassis.

resonance and 1:1 internal resonance, multiple-scale method was used to obtain a first-order approximate solution of the differential equations. Taking the parameters of the tracked ambulance for instance, the accuracy of the approximate solution was established compared with numerical results. The impact of the parameters on damping effect and motion stability was also investigated. Furthermore, the theoretical bases for improving efficiency of the damping system were put forward.

\section{Damping System Physical Model}

The tracked ambulance damping system is shown in Figure 1. The damping system is mainly constituted of rubber damping shock absorber and zero stiffness damper. The linear model is used to describe the stiffness and damping of the rubber damping shock absorber. For zero stiffness damper, the damping is described by a linear model, and stiffness is described by the positive and negative stiffness parallel model [7], shown in Figure 2.

The stiffness, the original length, and the initial deformation of horizontal spring, in Figure 1, are defined as $k, L$, and $\lambda$. $k_{0}$ stands for the stiffness of the vertical spring. The vertical elastic restoring force of the model can be expressed in the form

$$
f(x)=k_{0} x-k\left[x-\frac{L-\lambda}{\sqrt{1-(x / L)^{2}}} \frac{x}{L}\right] .
$$

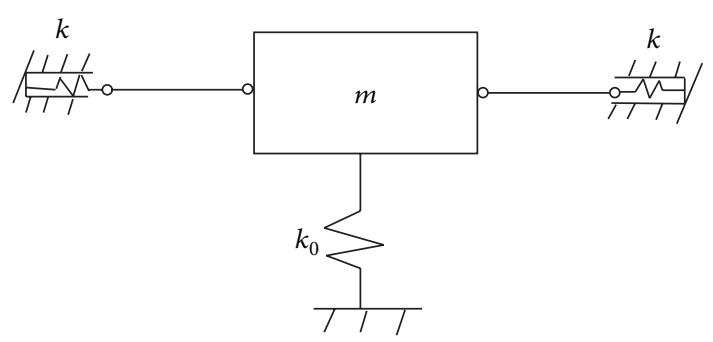

Figure 2: Positive and negative stiffness parallel model.

By using the Taylor series, a second-order expansion is sought in the form

$$
\frac{1}{\sqrt{1-(x / L)^{2}}}=1+\frac{1}{2}\left(\frac{x}{L}\right)^{2}+\frac{13}{24}\left(\frac{x}{L}\right)^{4}+\cdots .
$$

Substituting the first two terms into (1) results in

$$
f(x)=\left(k_{0}-\frac{k \lambda}{L}\right) x+\frac{L-\lambda}{L^{3}} \frac{k}{2} x^{3} \cdots .
$$

Therefore, the restoring force of zero stiffness damper can be expressed in the form

$$
f(z)=K_{s} x+\beta K_{s} x^{3} \cdots,
$$

where $K_{s}=\left(k_{0}-(k \lambda / L)\right), \beta K_{s}=\left((L-\lambda) / L^{3}\right)(k / 2)$, and $\beta$ is a small parameter.

According to the occupant of the vehicle ride (lying) comfort evaluation standards, occupant comfort is mainly affected by the vertical vibration acceleration. Neglecting the other two directions of vibration, a 2-DOF model of the tracked ambulance damping system is shown in Figure 3, including the following:

$M_{1}$ : the quality of stretcher and decubital body;

$M_{2}$ : the quality of carriage;

$K_{s}$ : the stiffness of zero stiffness damper;

$C_{1}$ : the damping of zero stiffness damper;

$K_{2}$ : the stiffness of rubber damping shock absorber;

$\mathrm{C}_{2}$ : the damping of rubber damping shock absorber;

$x_{1}, x_{2}$, and $x_{3}$ : stretcher base displacement, carriage displacement, chassis displacement.

The differential equations describing the motion of the damping system are

$$
\begin{gathered}
M_{1} \ddot{x}_{1}+C_{1}\left(\dot{x}_{1}-\dot{x}_{2}\right)+K_{s}\left(x_{1}-x_{2}\right)+\beta K_{s}\left(x_{1}-x_{2}\right)^{3}=0, \\
M_{2} \ddot{x}_{2}-C_{1}\left(\dot{x}_{1}-\dot{x}_{2}\right)-K_{s}\left(x_{1}-x_{2}\right)-\beta K_{s}\left(x_{1}-x_{2}\right)^{3} \\
+C_{2}\left(\dot{x}_{2}-\dot{x}_{3}\right)+K_{2}\left(x_{2}-x_{3}\right)=0 .
\end{gathered}
$$




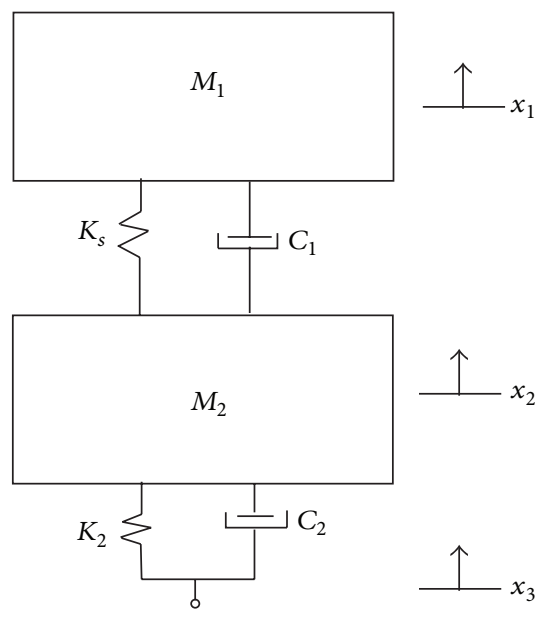

FIgURE 3: The 2-DOF damping system.

We rewrite (5) as

$$
\begin{gathered}
\ddot{x}_{1}+\omega_{1}^{2} x_{1}=l_{1} x_{2}-2 u_{1} \dot{x}_{1}+2 u_{1} \dot{x}_{2}-b_{1}\left(x_{1}-x_{2}\right)^{3}, \\
\ddot{x}_{2}+\omega_{2}^{2} x_{2}=f \cos \Omega t+2 u_{2} \dot{x}_{1}-2 u_{3} \dot{x}_{2}+l_{2} x_{1}+b_{2}\left(x_{1}-x_{2}\right)^{3},
\end{gathered}
$$

where

$$
\begin{gathered}
\omega_{1}^{2}=\frac{K_{s}}{M_{1}}, \quad l_{1}=\frac{K_{s}}{M_{1}}, \quad 2 u_{1}=\frac{C_{1}}{M_{1}}, \quad b_{1}=\frac{\beta K_{s}}{M_{1}}, \\
2 u_{2}=\frac{C_{1}}{M_{2}}, \quad \omega_{2}^{2}=\frac{K_{2}+K_{s}}{M_{2}}, \quad f \cos \Omega t=K_{2} x_{3}+C_{2} \dot{x}_{3}, \\
2 u_{3}=\frac{C_{1}+C_{2}}{M_{2}}, \quad l_{2}=\frac{K_{s}}{M_{2}}, \quad b_{2}=\frac{\beta K_{s}}{M_{2}} .
\end{gathered}
$$

\section{Linear Analysis}

Ignoring the impact of nonlinear terms, the nonlinear equations (5) are changed into linear equations as follows:

$$
\begin{gathered}
M_{1} \ddot{x}_{1}+C_{1}\left(\dot{x}_{1}-\dot{x}_{2}\right)+K_{s}\left(x_{1}-x_{2}\right)=0, \\
M_{2} \ddot{x}_{2}-C_{1}\left(\dot{x}_{1}-\dot{x}_{2}\right)-K_{s}\left(x_{1}-x_{2}\right)+C_{2}\left(\dot{x}_{2}-\dot{x}_{3}\right) \\
+K_{2}\left(x_{2}-x_{3}\right)=0 .
\end{gathered}
$$

By making the substitution,

$$
\begin{gathered}
x_{3}=e^{i \omega t}, \\
x_{1}(t)=H(\omega)_{x_{1}-x_{3}} e^{i \omega t}, \\
\dot{x}_{1}(t)=i \omega H(\omega)_{x_{1}-x_{3}} e^{i \omega t}, \\
\ddot{x}_{1}(t)=-\omega^{2} H(\omega)_{x_{1}-x_{3}} e^{i \omega t}, \\
\dot{x}_{3}=i \omega e^{i \omega t},
\end{gathered}
$$

$$
\begin{gathered}
x_{2}(t)=H(\omega)_{x_{2}-x_{3}} e^{i \omega t}, \\
\dot{x}_{2}(t)=i \omega H(\omega)_{x_{2}-x_{3}} e^{i \omega t}, \\
\ddot{x}_{2}(t)=-\omega^{2} H(\omega)_{x_{2}-x_{3}} e^{i \omega t} .
\end{gathered}
$$

Equations (8) become

$$
\begin{gathered}
\left(-\omega^{2} M_{1}+i \omega C_{1}+K_{s}\right) H(\omega)_{x_{1}-x_{3}}=\left(i \omega C_{1}+K_{s}\right) H(\omega)_{x_{2}-x_{3}} \\
\left(-\omega^{2} M_{2}+i \omega C_{1}+K_{s}+K_{2}+i \omega C_{2}\right) H(\omega)_{x_{2}-x_{3}} \\
=\left(i \omega C_{1}+K_{s}\right) H(\omega)_{x_{1}-x_{3}}+K_{2}+i \omega C_{2} .
\end{gathered}
$$

Setting $B_{1}=i \omega C_{1}+K_{s}, B_{2}=-\omega^{2} M_{1}+i \omega C_{1}+K_{s}$, and $B_{3}=-\omega^{2} M_{2}+i \omega C_{1}+K_{s}+K_{2}+i C_{2} \omega$, we obtain

$$
\begin{aligned}
& H(\omega)_{x_{1}-x_{3}}=\frac{B_{1}\left(K_{2}+i \omega C_{2}\right)}{B_{3} B_{2}-B_{1}^{2}}, \\
& H(\omega)_{x_{2}-x_{3}}=\frac{B_{2}\left(K_{2}+i \omega C_{2}\right)}{B_{3} B_{2}-B_{1}^{2}} .
\end{aligned}
$$

The steady-state amplitude from (11) is

$$
\begin{gathered}
|H(\omega)|_{x_{1}-x_{3}} \\
=\gamma\left[\frac{\left(1-4 \lambda^{2} \xi_{1} \xi_{2} \sqrt{1 / \gamma u}\right)^{2}+4 \lambda^{2}\left(\xi_{2} \sqrt{1 / \gamma u}+\lambda \xi_{1}\right)^{2}}{\Delta}\right]^{1 / 2}, \\
|H(\omega)|_{x_{2}-x_{3}} \\
=\gamma\left[\left(\left(1-\lambda^{2}-4 \lambda^{2} \xi_{1} \xi_{2} \sqrt{1 / \gamma u}\right)^{2}\right.\right. \\
\left.\quad+4 \lambda^{2}\left(-\lambda^{2} \xi_{2} \sqrt{1 / \gamma u}+\xi_{2} \sqrt{1 / \gamma u}+\xi_{1}\right)^{2}\right) \\
\left.\times(\Delta)^{-1}\right]^{1 / 2},
\end{gathered}
$$

where

$$
\begin{gathered}
\Delta=\left[\left(-\lambda^{2}+1\right)\left(1+\gamma-\frac{\lambda^{2}}{u}\right)-4 \lambda^{2} \xi_{1} \xi_{2} \sqrt{\frac{\gamma}{u}}-1\right]^{2} \\
+4 \lambda^{2} \xi_{1}^{2}\left[\gamma-\left(1+\frac{1}{u}\right) \lambda^{2}+\frac{\xi_{2}}{\xi_{1}} \sqrt{\frac{\gamma}{u}}\left(1-\lambda^{2}\right)\right]^{2}, \\
\xi_{1}=\frac{C_{1}}{2 \sqrt{M_{1} K_{s}}}, \quad \xi_{2}=\frac{C_{2}}{2 \sqrt{M_{2} K_{2}}}, \quad u=\frac{M_{1}}{M_{2}}, \\
\lambda=\frac{\omega}{\omega_{1}}, \quad \omega_{1}=\sqrt{\frac{K_{s}}{M_{1}}}, \quad \gamma=\frac{K_{2}}{K_{s}}, \quad \omega_{2}=\sqrt{\frac{K_{2}}{M_{2}}} .
\end{gathered}
$$




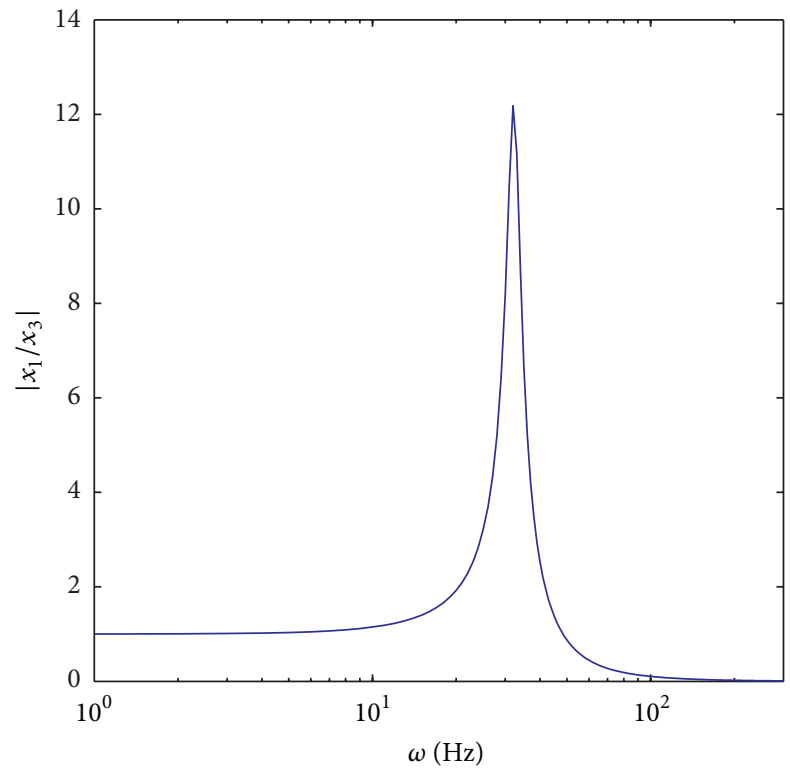

(a)

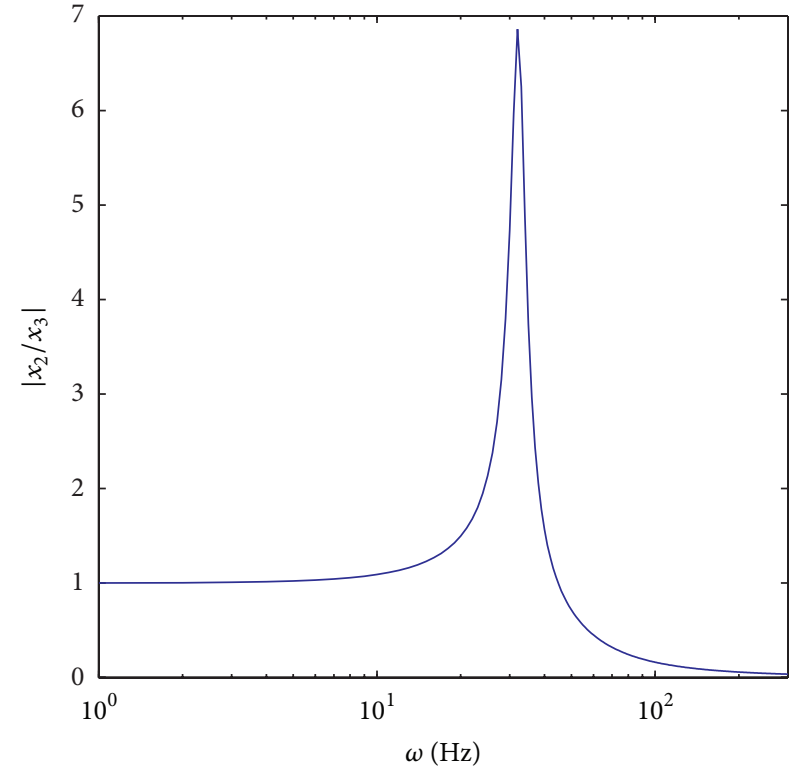

(b)

Figure 4: Amplitude-frequency curves.

The amplitude-frequency curves are plotted with the parameters of a tracked ambulance. The parameters of the tracked ambulance are $M_{1}=180 \mathrm{~kg}, M_{2}=2000 \mathrm{~kg}, K_{s}=$ $217582 \mathrm{~N} / \mathrm{m}, C_{1}=4200 \mathrm{~N} \cdot \mathrm{s} / \mathrm{m}, f=1500 \mathrm{~N}, \beta=0.1$, $K_{2}=2200000 \mathrm{~N} / \mathrm{m}$, and $C_{2}=19540 \mathrm{~N} \cdot \mathrm{s} / \mathrm{m}$. The amplitudefrequency curves are shown in Figure 4. Although the model is 2-DOF, there is only one resonance peak. This is because $\omega_{1}^{2} \approx \omega_{2}^{2}$.

\section{Perturbation Analysis}

Utilizing multiscale method, the response of damping system is solved. The new independent time scales

$$
T_{n}=\varepsilon^{n} t, \quad n=0,1, \ldots
$$

are introduced, where $\varepsilon$ represents a small positive parameter and $T_{n}, n=0,1, \ldots$ are "slow" time scales which capture the response due to the nonlinearities, damping, and external excitation. And we note that

$$
\begin{gathered}
\frac{d}{d t}=D_{0}+\varepsilon D_{1}+\cdots, \\
\frac{d^{2}}{d t^{2}}=D_{0}^{2}+2 \varepsilon D_{0} D_{1}+\varepsilon^{2}\left(D_{1}^{2}+2 D_{0} D_{2}\right)+\cdots,
\end{gathered}
$$

where $D_{n}=\partial / \partial T_{n}, n=0,1, \ldots$. We expand the timedependent variables $x_{1}$ and $x_{2}$ in powers of $\varepsilon$ as

$$
\begin{aligned}
& x_{1}=x_{11}\left(T_{0}, T_{1}, \ldots\right)+\varepsilon x_{12}\left(T_{0}, T_{1}, \ldots\right)+\cdots, \\
& x_{2}=x_{21}\left(T_{0}, T_{1}, \ldots\right)+\varepsilon x_{22}\left(T_{0}, T_{1}, \ldots\right)+\cdots .
\end{aligned}
$$

Then we substitute (15)-(16) into (6) and obtain the following equations.
$\operatorname{Order}\left(\varepsilon^{0}\right)$ :

$$
\begin{aligned}
& D_{0}^{2} x_{11}+\omega_{1}^{2} x_{11}=0 \\
& D_{0}^{2} x_{21}+\omega_{2}^{2} x_{21}=0 .
\end{aligned}
$$

$\operatorname{Order}\left(\varepsilon^{1}\right)$ :

$$
\begin{aligned}
D_{0}^{2} x_{12}+\omega_{1}^{2} x_{12}= & -2 D_{0}\left(D_{1} x_{11}+u_{1} x_{11}-u_{1} x_{21}\right)+l_{1} x_{21} \\
& -b_{1} x_{11}^{3}+3 b_{1} x_{11}^{2} x_{21}-3 b_{1} x_{11} x_{21}^{2}+b_{1} x_{21}^{3}, \\
D_{0}^{2} x_{22}+\omega_{2}^{2} x_{22}= & -2 D_{0}\left(D_{1} x_{21}-u_{2} x_{11}+u_{3} x_{21}\right) \\
& +l_{2} x_{11}+b_{2} x_{11}^{3}-3 b_{2} x_{11}^{2} x_{21} \\
& +3 b_{2} x_{11} x_{21}^{2}-b_{2} x_{21}^{3}+f \cos \left(\Omega T_{0}\right) .
\end{aligned}
$$

The solution of (17) can be expressed as

$$
\begin{aligned}
& x_{11}=A_{1}\left(T_{1}\right) \exp \left(i \omega_{1} T_{0}\right)+c c \\
& x_{21}=A_{2}\left(T_{1}\right) \exp \left(i \omega_{2} T_{0}\right)+c c .
\end{aligned}
$$

To express 1:1 internal resonance and the nearness of the excitation frequency to the first-order natural frequency, we introduce two detuning parameters $\sigma_{1}$ and $\sigma_{2}$ defined by $\omega_{2}=\omega_{1}+\varepsilon \sigma_{1}, \Omega=\omega_{1}+\varepsilon \sigma_{2}$. Substitution of (19) and $\omega_{2}=\omega_{1}+\varepsilon \sigma_{1}, \Omega=\omega_{1}+\varepsilon \sigma_{2}$ into (18) leads to secular terms. 
By eliminating these secular terms, the state equations are obtained as follows:

$$
\begin{aligned}
& -2 A_{1}^{\prime} i \omega_{1}-2 u_{1} A_{1} i \omega_{1}+2 u_{1} i \omega_{2} A_{2} \exp \left(i \sigma T_{0}\right) \\
& +l_{1} A_{2} \exp \left(i \sigma T_{0}\right)-3 b_{1} A_{1}^{2} \bar{A}_{1}+3 b_{1} A_{2}^{2} \bar{A}_{2} \exp \left(i \sigma T_{0}\right) \\
& +3 b_{1} A_{1}^{2} \bar{A}_{2} \exp \left(-i \sigma T_{0}\right)+6 b_{1} A_{1} \bar{A}_{1} A_{2} \exp \left(i \sigma T_{0}\right) \\
& \quad-6 b_{1} A_{1} \bar{A}_{2} A_{2}-3 b_{1} \bar{A}_{1} A_{2}^{2} \exp \left(2 i \sigma T_{0}\right)=0 \\
& -2 A_{2}^{\prime} i \omega_{2}-2 u_{3} A_{2} i \omega_{2}+2 u_{2} A_{1} i \omega_{1} \exp \left(-i \sigma T_{0}\right) \\
& +l_{2} A_{1} \exp \left(-i \sigma T_{0}\right)+3 b_{2} A_{1}^{2} \bar{A}_{1} \exp \left(-i \sigma T_{0}\right) \\
& -3 b_{2} A_{2}^{2} \bar{A}_{2}-3 b_{2} A_{1}^{2} \bar{A}_{2} \exp \left(-2 i \sigma T_{0}\right)-6 b_{2} A_{1} \bar{A}_{1} A_{2} \\
& +6 b_{2} A_{1} \bar{A}_{2} A_{2} \exp \left(-i \sigma T_{0}\right)+3 b_{2} \bar{A}_{1} A_{2}^{2} \exp \left(i \sigma T_{0}\right) \\
& +\frac{1}{2} f \exp \left(i \sigma_{2} T_{1}-i \sigma_{1} T_{1}\right)=0
\end{aligned}
$$

where $A_{n}^{\prime}=D_{1} A_{n}, \sigma=\varepsilon \sigma_{1}$. Introducing the polar form

$$
A_{n}=\frac{1}{2} a_{n} \exp \left(i \theta_{n}\right), \quad n=1,2
$$

into (20) and separating the equation into real and imaginary parts result in the following four state equations:

$$
\begin{aligned}
-a_{1}^{\prime} \omega_{1}-u_{1} a_{1} \omega_{1}+u_{1} \omega_{2} a_{2} \cos \gamma+\frac{1}{2} l_{1} a_{2} \sin \gamma+\frac{3}{8} b_{1} a_{2}^{3} \sin \gamma \\
+\frac{3}{8} b_{1} a_{1}^{2} a_{2} \sin \gamma-\frac{3}{8} b_{1} a_{1} a_{2}^{2} \sin 2 \gamma=0 \\
a_{1} \theta_{1}^{\prime} \omega_{1}-u_{1} \omega_{2} a_{2} \sin \gamma+\frac{1}{2} l_{1} a_{2} \cos \gamma-\frac{3}{8} b_{1} a_{1}^{3}+\frac{3}{8} b_{1} a_{2}^{3} \cos \gamma \\
+\frac{9}{8} b_{1} a_{1}^{2} a_{2} \cos \gamma-\frac{3}{4} b_{1} a_{1} a_{2}^{2}-\frac{3}{8} b_{1} a_{1} a_{2}^{2} \cos 2 \gamma=0, \\
-a_{2}^{\prime} \omega_{2}-u_{3} a_{2} \omega_{2}+u_{2} a_{1} \omega_{1} \cos \gamma-\frac{1}{2} l_{2} a_{1} \sin \gamma-\frac{3}{8} b_{2} a_{1}^{3} \sin \gamma \\
+\frac{3}{8} b_{2} a_{1}^{2} a_{2} \sin 2 \gamma-\frac{3}{8} b_{2} a_{1} a_{2}^{2} \sin \gamma+\frac{1}{2} f \sin \varphi=0, \\
a_{2} \omega_{2} \theta_{2}^{\prime}+u_{2} a_{1} \omega_{1} \sin \gamma+\frac{1}{2} l_{2} a_{1} \cos \gamma+\frac{3}{8} b_{2} a_{1}^{3} \cos \gamma \\
-\frac{3}{4} b_{2} a_{1}^{2} a_{2}-\frac{3}{8} b_{2} a_{1}^{2} a_{2} \cos 2 \gamma+\frac{9}{8} b_{2} a_{1} a_{2}^{2} \cos \gamma-\frac{3}{8} b_{2} a_{2}^{3} \\
+\frac{1}{2} f \cos \varphi=0,
\end{aligned}
$$

where $\gamma=\sigma T_{0}+\theta_{2}-\theta_{1}, \varphi=\sigma_{2} T_{1}-\sigma_{1} T_{1}-\theta_{2}$. At steady state, $a_{n}^{\prime}=\theta_{n}^{\prime}=0$ and the average equations (22) become

$$
\begin{gathered}
-u_{1} a_{1} \omega_{1}+u_{1} \omega_{2} a_{2} \cos \gamma-\frac{3}{8} b_{1} a_{1} a_{2}^{2} \sin 2 \gamma \\
+\frac{3}{8} b_{1} a_{1}^{2} a_{2} \sin \gamma+\frac{3}{8} b_{1} a_{2}^{3} \sin \gamma+\frac{1}{2} l_{1} a_{2} \sin \gamma=0 \\
a_{1} \sigma_{2} \omega_{1}-u_{1} \omega_{2} a_{2} \sin \gamma+\frac{1}{2} l_{1} a_{2} \cos \gamma-\frac{3}{8} b_{1} a_{1}^{3}+\frac{3}{8} b_{1} a_{2}^{3} \cos \gamma \\
+\frac{9}{8} b_{1} a_{1}^{2} a_{2} \cos \gamma-\frac{3}{4} b_{1} a_{1} a_{2}^{2}-\frac{3}{8} b_{1} a_{1} a_{2}^{2} \cos 2 \gamma=0 \\
-u_{3} a_{2} \omega_{2}+u_{2} a_{1} \omega_{1} \cos \gamma-\frac{3}{8} b_{2} a_{1}^{3} \sin \gamma+\frac{3}{8} b_{2} a_{1}^{2} a_{2} \sin 2 \gamma \\
-\frac{1}{2} l_{2} a_{1} \sin \gamma-\frac{3}{8} b_{2} a_{1} a_{2}^{2} \sin \gamma+\frac{1}{2} f \sin \varphi=0 \\
a_{2} \omega_{2}\left(\sigma_{2}-\sigma_{1}\right)+u_{2} a_{1} \omega_{1} \sin \gamma+\frac{1}{2} l_{2} a_{1} \cos \gamma+\frac{3}{8} b_{2} a_{1}^{3} \cos \gamma \\
-\frac{3}{8} b_{2} a_{2}^{3}-\frac{3}{8} b_{2} a_{1}^{2} a_{2} \cos 2 \gamma-\frac{3}{4} b_{2} a_{1}^{2} a_{2}+\frac{9}{8} b_{2} a_{1} a_{2}^{2} \cos \gamma \\
+\frac{1}{2} f \cos \varphi=0
\end{gathered}
$$

which provide the steady-state amplitudes and phases.

\section{Simulation Analysis}

The numerical simulation is to analyze the relationship between the vibration amplitudes of the stretcher base and carriage and the parameters of tracked ambulance. To establish the accuracy of the average equations, simulation analysis is performed for the parameters shown previously [8]. For $\omega_{1}=\omega_{2}=34.8 \mathrm{rad} / \mathrm{s}$, the $1: 1$ resonance may occur. In Figure 5, we show the comparison between numerical solutions, obtained by the Runge-Kutta method, and perturbation solutions.

In Figure 5, the trend and resonance position of perturbation solutions and numerical solutions are the same, but the amplitudes are different. This is because we only use the firstorder approximation, and it does not affect our qualitative analysis of the dynamic behavior of the system. The damping system amplitude-frequency curve is similar to the linear results, where jump phenomenon does not occur. Figures 610 show the impact of the tracked ambulance's parameters on the damping effect, where $a_{1}$ and $a_{2}$ represent the vibrating amplitudes of the carriage and the stretcher base.

As can be seen from Figure 6, when the amplitude of the exciting force becomes bigger, the vibration amplitudes of stretcher base and carriage all become bigger.

Figures 7 and 8 clearly show that the damping of zero stiffness damper has a great impact on the vibration amplitude of stretcher base, but little on the vibration amplitude of carriage and the damping of rubber damping shock absorber 


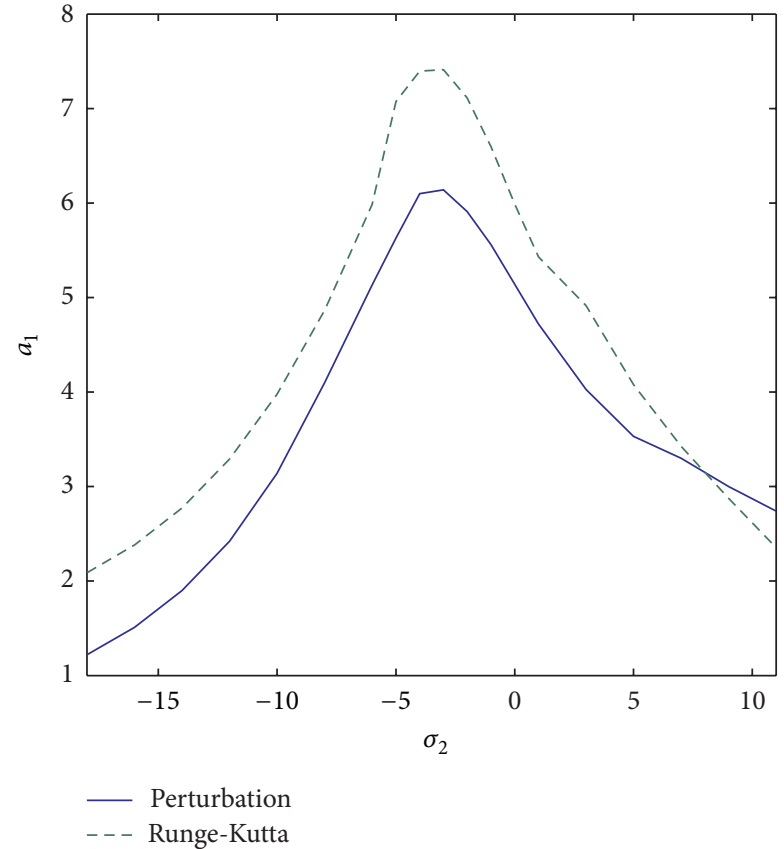

(a)

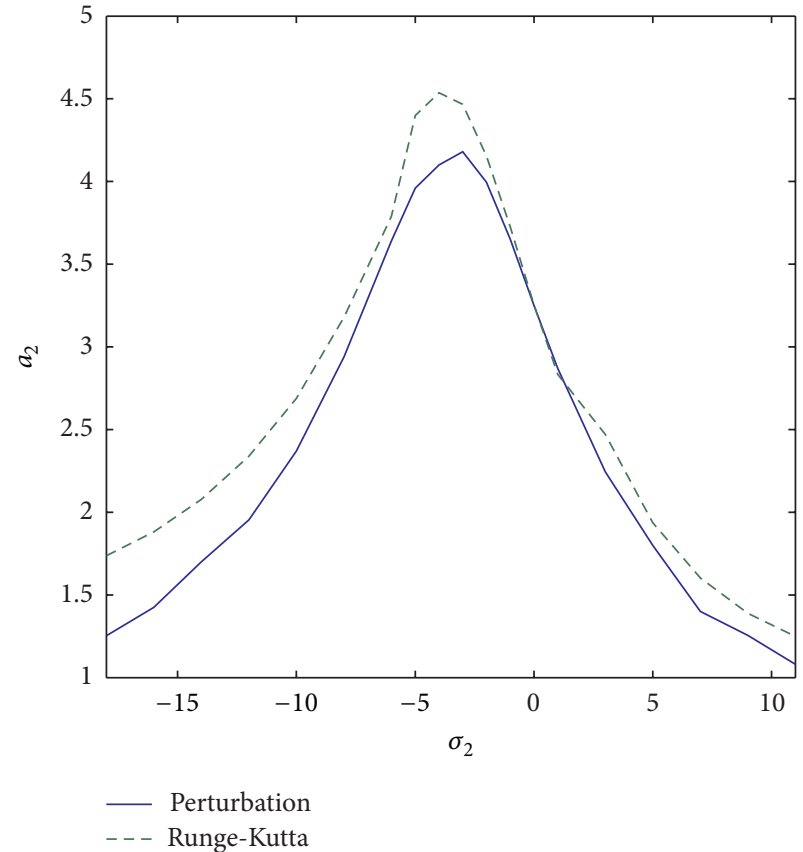

(b)

FIgURE 5: Amplitude-frequency curve.

has a great impact on the vibration amplitudes of stretcher base and carriage. The damping is greater and the amplitude is smaller. Hence, increasing the damping of rubber damping shock absorber, to some degree, is more effective reducing vibration.

Figures 9 and 10 show that the stiffness of zero stiffness damper only has a major impact on the vibration amplitude of stretcher base, and the stiffness of rubber damping shock absorber only has a major impact on the vibration amplitude of carriage on the premise of meeting 1:1 internal resonance roughly. However, both stiffness of zero stiffness damper and rubber damping shock absorber affect the resonance frequency. Increasing the stiffness of zero stiffness damper or decreasing the stiffness of rubber damping shock absorber can increase the resonance frequency, which is helpful in decreasing vehicle vibration [9]. With comprehensive comparison of Figures 7-10, damping has a great influence on the amplitude of vibration, and stiffness has a great impact on the resonance frequency.

\section{Stability Analysis}

To analyze the stability of the system in the primary resonance, we need to convert the average equations in polar form into a rectangular form by introducing [10-14] $p_{1}=$ $a_{1} \cos (\gamma+\varphi), q_{1}=a_{1} \sin (\gamma+\varphi), p_{2}=a_{2} \cos \varphi$, and $q_{2}=$ $a_{2} \sin \varphi$ resulting in

$$
\dot{p}_{1}=-u_{1} p_{1}+u_{1} p_{2}-\frac{l_{1}}{2 \omega_{1}} q_{2}-\frac{3}{8 \omega_{1}} b_{1}\left(p_{2}^{2}+q_{2}^{2}\right) q_{2}
$$

$$
\begin{aligned}
& -\sigma_{2} q_{1}-\frac{3}{8 \omega_{1}} b_{1}\left(p_{1}^{2}+q_{1}^{2}\right) q_{2} \\
& -\frac{3}{8 \omega_{1}} b_{1}\left(q_{1} p_{2}^{2}-q_{2}^{2} q_{1}-2 p_{1} p_{2} q_{2}\right) \\
& -\frac{3}{4 \omega_{1}} b_{1}\left(p_{1} p_{2}+q_{1} q_{2}\right) q_{1}+\frac{3}{4 \omega_{1}} b_{1}\left(p_{2}^{2}+q_{2}^{2}\right) q_{1} \\
& +\frac{3}{8 \omega_{1}} b_{1}\left(p_{1}^{2}+q_{1}^{2}\right) q_{1}, \\
\dot{p}_{2}= & -u_{3} p_{2}+u_{2} p_{1}-\frac{l_{2}}{2 \omega_{2}} q_{1}-\frac{3}{8 \omega_{2}} b_{2}\left(p_{1}^{2}+q_{1}^{2}\right) q_{1} \\
& -\left(\sigma_{2}-\sigma_{1}\right) q_{2}+\frac{3}{8 \omega_{2}} b_{2}\left(2 p_{1} q_{1} p_{2}-p_{1}^{2} q_{2}+q_{1}^{2} q_{2}\right) \\
& -\frac{3}{8 \omega_{2}} b_{2}\left(p_{2}^{2}+q_{2}^{2}\right) q_{1}+\frac{3}{8 \omega_{2}} b_{2}\left(p_{2}^{2}+q_{2}^{2}\right) q_{2} \\
& +\frac{3}{4 \omega_{2}} b_{2}\left(p_{1}^{2}+q_{1}^{2}\right) q_{2}-\frac{3}{4 \omega_{2}} b_{2}\left(p_{1} p_{2}+q_{1} q_{2}\right) q_{2}, \\
\dot{q}_{1}= & -u_{1} q_{1}+u_{1} q_{2}+\frac{l_{1}}{2 \omega_{1}} p_{2}+\frac{3}{8 \omega_{1}} b_{1}\left(p_{2}^{2}+q_{2}^{2}\right) p_{2} \\
& +\sigma_{2} p_{1}+\frac{3}{8 \omega_{1}} b_{1}\left(p_{1}^{2}+q_{1}^{2}\right) p_{2} \\
& -\frac{3}{8 \omega_{1}} b_{1}\left(p_{1} p_{2}^{2}-q_{2}^{2} p_{1}+2 q_{1} p_{2} q_{2}\right) \\
& +\frac{3}{4 \omega_{1}} b_{1}\left(p_{1} p_{2}+q_{1} q_{2}\right) p_{1}-\frac{3}{4 \omega_{1}} b_{1}\left(p_{2}^{2}+q_{2}^{2}\right) p_{1}
\end{aligned}
$$



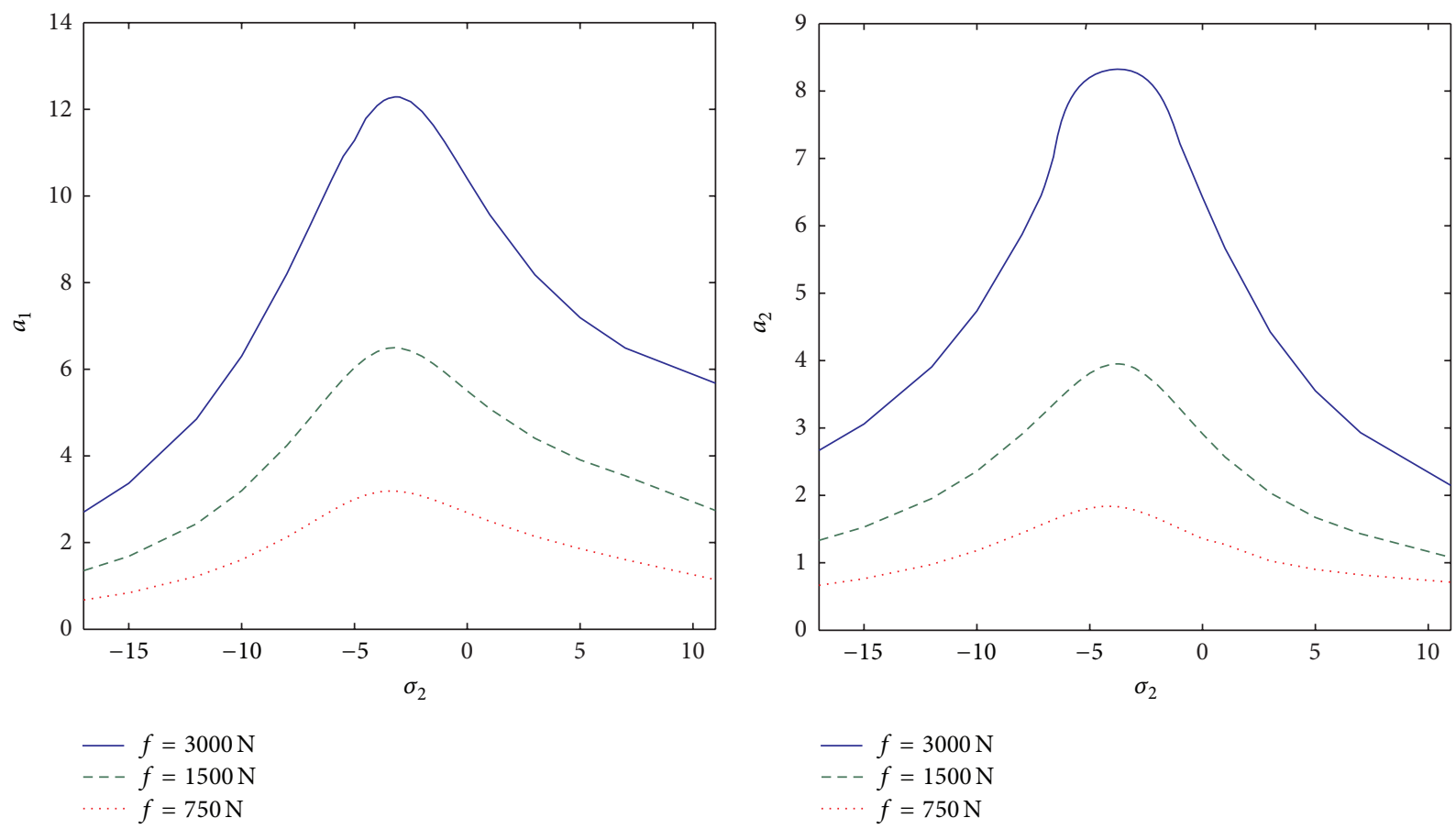

(a)

(b)

FIgUre 6: The influence of the exciting force.
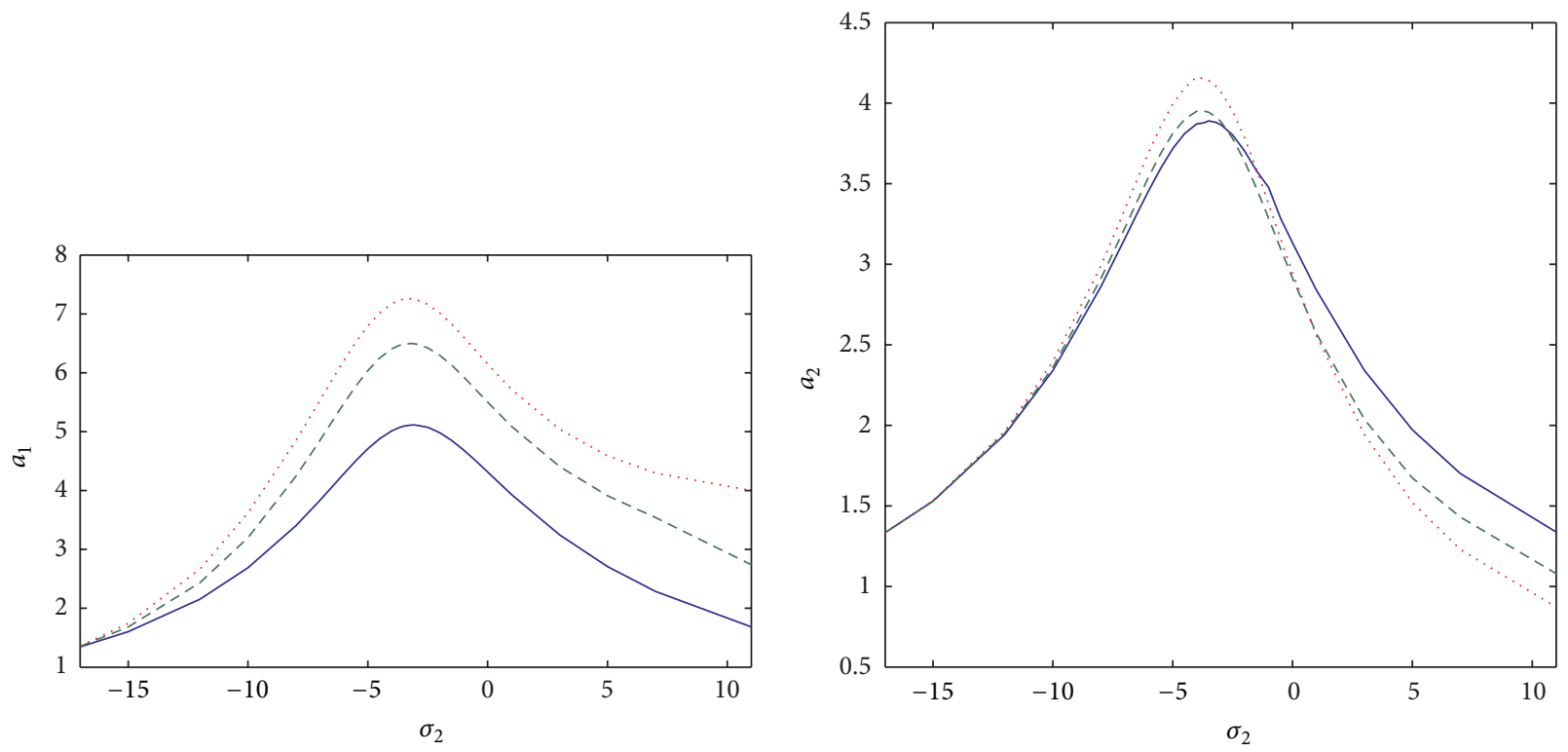

$\begin{aligned} \ldots C_{1} & =8400 \mathrm{~N} \cdot \mathrm{s} / \mathrm{m} \\ --C_{1} & =4200 \mathrm{~N} \cdot \mathrm{s} / \mathrm{m} \\ -C_{1} & =2100 \mathrm{~N} \cdot \mathrm{s} / \mathrm{m}\end{aligned}$

$$
\begin{aligned}
& -C_{1}=8400 \mathrm{~N} \cdot \mathrm{s} / \mathrm{m} \\
& --C_{1}=4200 \mathrm{~N} \cdot \mathrm{s} / \mathrm{m} \\
& \ldots . C_{1}=2100 \mathrm{~N} \cdot \mathrm{s} / \mathrm{m}
\end{aligned}
$$

(a)

(b)

FIGURE 7: The influence of zero stiffness damper's damping. 

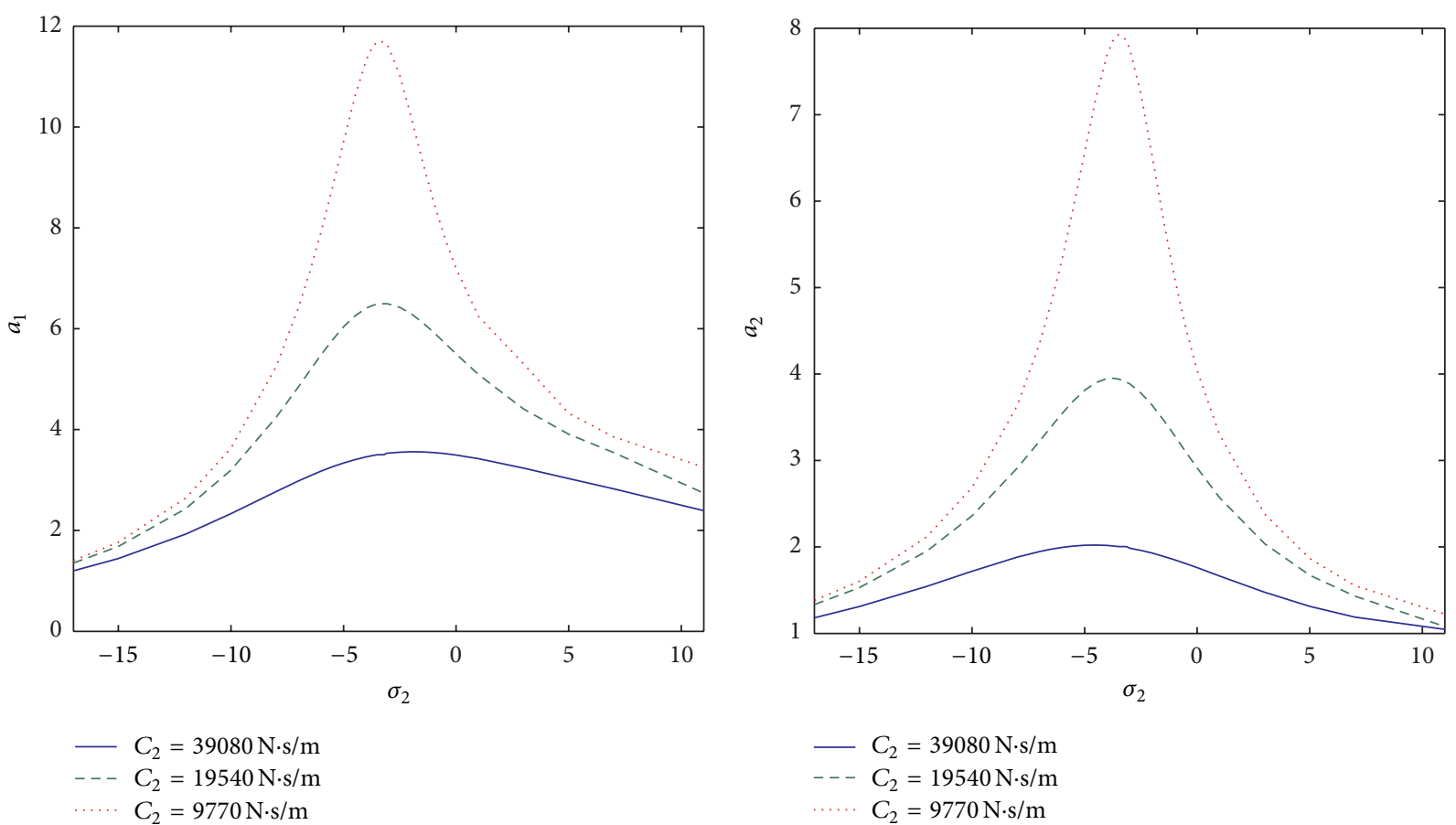

(a)

(b)

FIGURE 8: The influence of rubber damping shock absorber's damping.
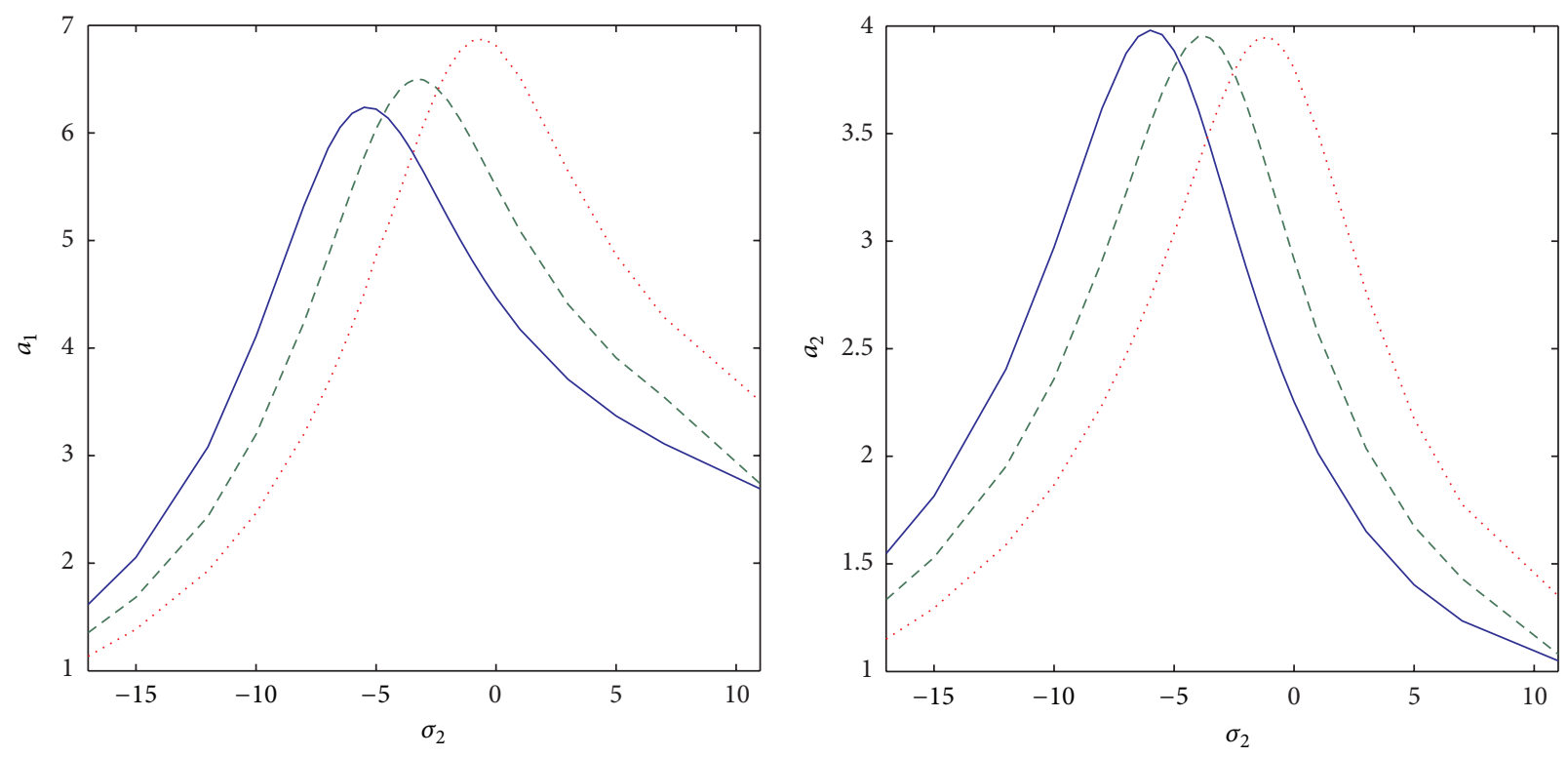

$\begin{aligned}-K_{s} & =246420 \mathrm{~N} / \mathrm{m} \\ ---K_{s} & =217582 \mathrm{~N} / \mathrm{m} \\ \cdots . . K_{s} & =184320 \mathrm{~N} / \mathrm{m}\end{aligned}$

$\begin{aligned}- & K_{s}=246420 \mathrm{~N} / \mathrm{m} \\ --- & K_{s}=217582 \mathrm{~N} / \mathrm{m}\end{aligned}$

…. $K_{s}=184320 \mathrm{~N} / \mathrm{m}$

(a)

Figure 9: The influence of zero stiffness damper's stiffness. 


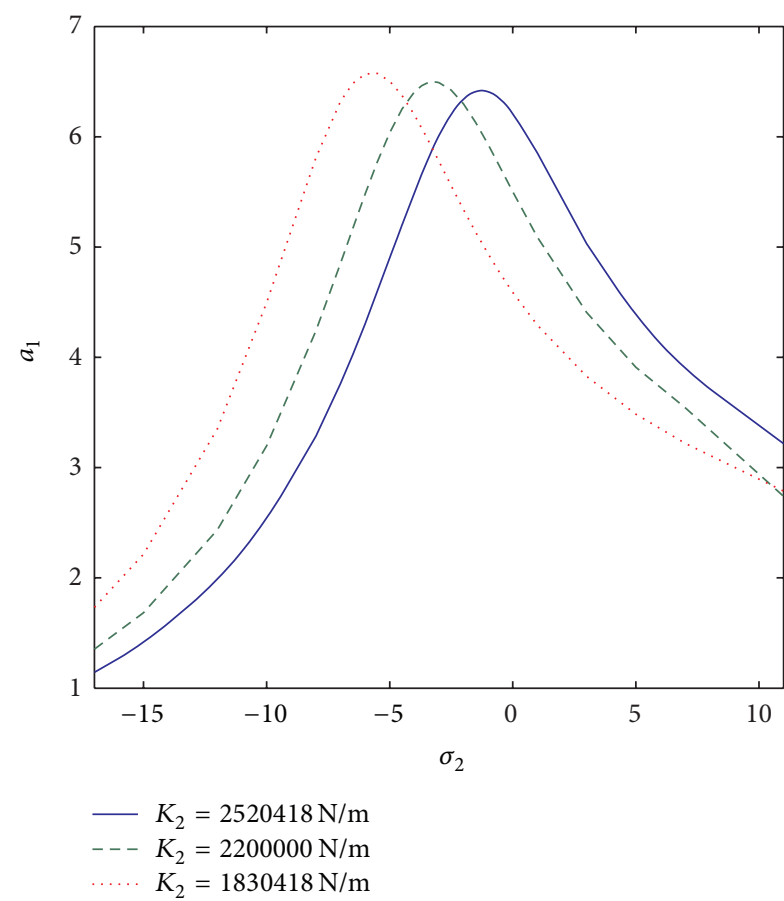

(a)

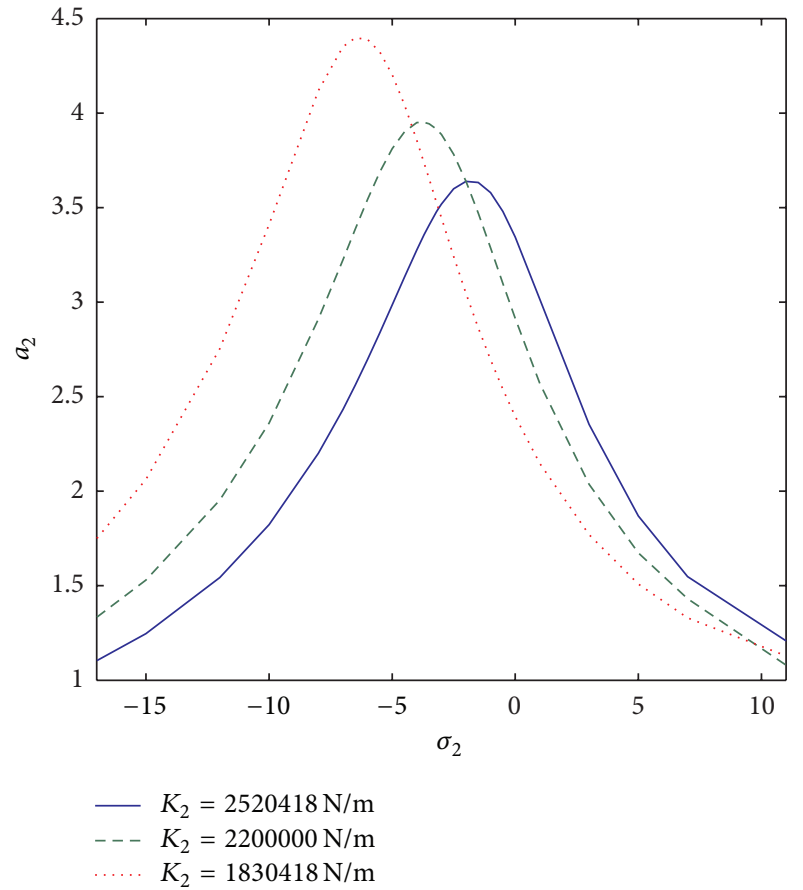

(b)

FIgURE 10: The influence of rubber damping shock absorber's stiffness.

$$
\begin{aligned}
& -\frac{3}{8 \omega_{1}} b_{1}\left(p_{1}^{2}+q_{1}^{2}\right) p_{1}, \\
\dot{q}_{2}= & -u_{3} q_{2}+u_{2} q_{1}+\frac{l_{2}}{2 \omega_{2}} p_{1} \\
& +\frac{3}{8 \omega_{2}} b_{2}\left(p_{1}^{2}+q_{1}^{2}\right) p_{1}+\frac{f}{2 \omega_{2}} \\
& -\frac{3}{8 \omega_{2}} b_{2}\left(p_{1}^{2} q_{1}-q_{1}^{3}+2 p_{1} q_{1} q_{2}\right) \\
& +\frac{3}{8 \omega_{2}} b_{2}\left(p_{2}^{2}+q_{2}^{2}\right) p_{1}+\left(\sigma_{2}-\sigma_{1}\right) p_{2} \\
& -\frac{3}{8 \omega_{2}} b_{2}\left(p_{2}^{2}+q_{2}^{2}\right) p_{2}-\frac{3}{4 \omega_{2}} b_{2}\left(p_{1}^{2}+q_{1}^{2}\right) p_{2} \\
& +\frac{3}{4 \omega_{2}} b_{2}\left(p_{1} p_{2}+q_{1} q_{2}\right) p_{2},
\end{aligned}
$$

where the average equations become more complex and the exact analytical solution cannot be obtained. At steady state, $\dot{p}_{1}=0, \dot{p}_{2}=0, \dot{q}_{1}=0$, and $\dot{q}_{2}=0$, and we use the Newton Method to calculate the value of the equilibrium point of the average equations (22) by repeatedly changing the initial value of the equilibrium point. There are three sets of equilibrium points

$$
\phi_{1}=\{-3.9041,-1.2622,2.0696,1.8797\},
$$

$$
\begin{aligned}
& \phi_{2}=\{12.1124,7.7186,-13.7048,-10.4106\}, \\
& \phi_{3}=\{-10.9811,-6.7073,11.9741,8.9831\} .
\end{aligned}
$$

The stability of the system at the equilibrium point is governed by the eigenvalue of the Jacobian matrix of (27) based on the singularity theory. The eigenvalues are obtained as follows:

$$
\begin{aligned}
\lambda_{1}= & \{-13.5988+22.7426 i,-13.5988-22.7426 i, \\
& -4.0362+5.5237 i,-4.0362-5.5237 i\}, \\
\lambda_{2}= & \{-31.75+120.17 i, 30.18,-31.75-120.17 i,-1.95\}, \\
\lambda_{3}= & \{-28.95+103.88 i,-1.70,-28.95-103.88 i, 24.33\} .
\end{aligned}
$$

Equation (23) is the Jacobian matrix of (22) at equilibrium point, where the expressions of $n_{i j}(i=1, \ldots, 4, j=1, \ldots, 4)$ are given in the appendix:

$$
A=\left[\begin{array}{llll}
n_{11} & n_{12} & n_{13} & n_{14} \\
n_{21} & n_{22} & n_{23} & n_{24} \\
n_{31} & n_{32} & n_{33} & n_{34} \\
n_{41} & n_{42} & n_{43} & n_{44}
\end{array}\right] .
$$

After singularity analysis, the system is only stable in the first equilibrium point. Since there is only one stable equilibrium point, the jump phenomenon does not occur. The Runge-Kutta method is used to validate the singularity analysis. Figure 11 presents the final stable position of (22) at three equilibrium points. 


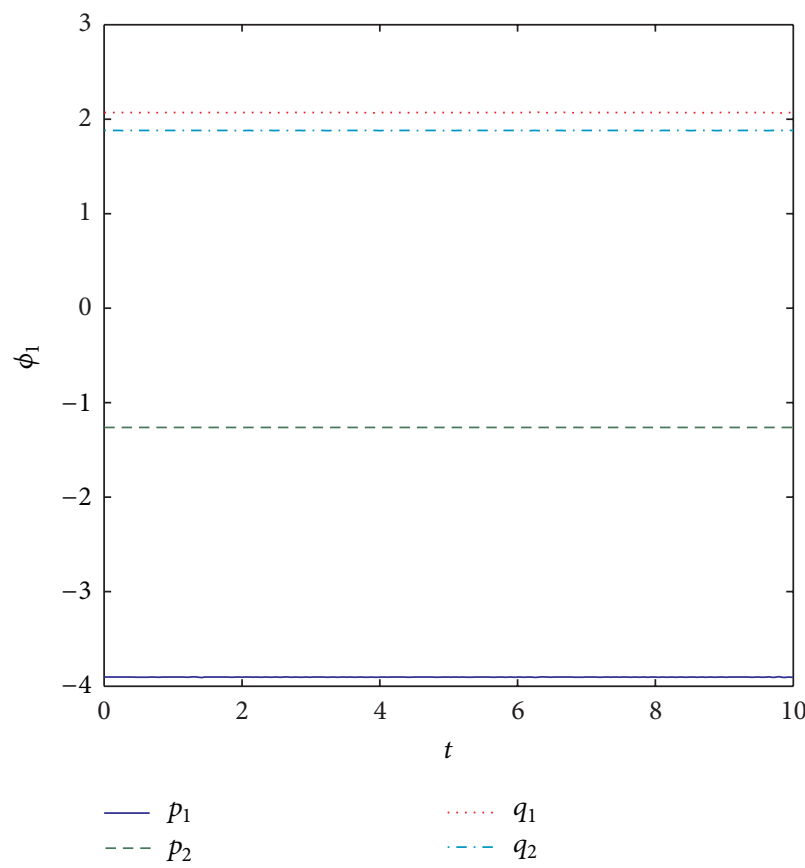

(a)

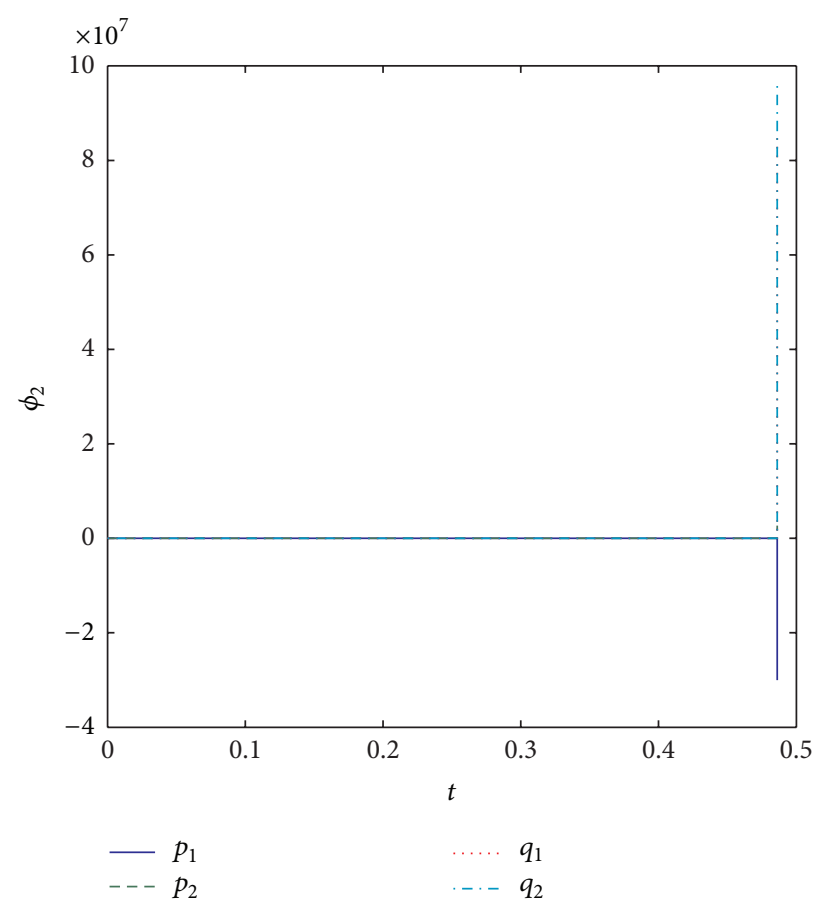

(b)

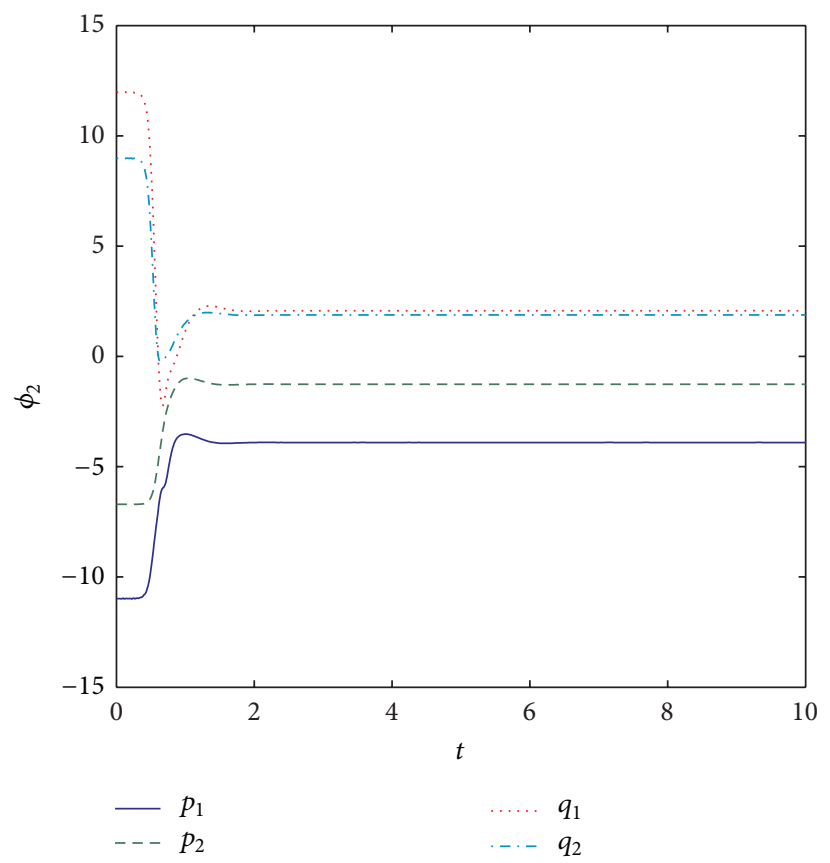

(c)

FIgURE 11: System stability location.

Figure 11 clearly illustrates that the system is only stable in the first equilibrium point and diverges to infinity (Figure 11(b)) or converges to the stable equilibrium point (Figure 11(c)) at an unstable equilibrium point. Therefore, the system is impossible to get a stable state in the unstable equilibrium point.

\section{Conclusion}

This paper established the dynamic model of a tracked ambulance damping system containing cubic nonlinear terms. We utilized a multiscale method to investigate the dynamic model and obtained the average equations. Average equations 
were corroborated with the actual parameters. The influence of damping system parameters on the damping effect as well as the stability of the damping system was analyzed. The result explained the reasons why there is no jump phenomenon. This analysis method is suitable for multidegree-of-freedom bearing motion system, particularly suitable for vehicles. The research results are valuable for the vehicle damping system design as well as forecast the damping system dynamic behavior.

\section{Appendix}

Consider

$$
\begin{aligned}
& n_{11}=-u_{1}-\frac{3 b_{1} q_{2} p_{1}}{4 \omega_{1}}+\frac{3 b_{1} q_{2} p_{2}}{4 \omega_{1}}-\frac{3 b_{1} q_{1} p_{2}}{4 \omega_{1}}+\frac{3 b_{1} q_{1} p_{1}}{4 \omega_{1}} \\
& n_{12}=u_{1}-\frac{3 b_{1} q_{2} p_{2}}{4 \omega_{1}}-\frac{3 b_{1}\left(2 q_{1} p_{2}-2 p_{1} q_{2}\right)}{8 \omega_{1}} \\
& -\frac{3 b_{1} q_{1} p_{1}}{4 \omega_{1}}+\frac{3 b_{1} q_{1} p_{2}}{2 \omega_{1}} \\
& n_{13}=-\frac{3 b_{1} q_{2} q_{1}}{2 \omega_{1}}-\frac{3 b_{1}\left(p_{2}^{2}-q_{2}^{2}\right)}{8 \omega_{1}}-\sigma_{2}-\frac{3 b_{1}\left(p_{1} p_{2}+q_{1} q_{2}\right)}{4 \omega_{1}} \\
& +\frac{3 b_{1}\left(p_{2}^{2}+q_{2}^{2}\right)}{4 \omega_{1}}+\frac{3 b_{1} q_{1}^{2}}{4 \omega_{1}}+\frac{3 b_{1}\left(p_{1}^{2}+q_{1}^{2}\right)}{8 \omega_{1}}, \\
& n_{14}=-\frac{l_{1}}{2 \omega_{1}}-\frac{3 b_{1} q_{2}^{2}}{4 \omega_{1}}-\frac{3 b_{1}\left(p_{2}^{2}+q_{2}^{2}\right)}{8 \omega_{1}}-\frac{3 b_{1}\left(p_{1}^{2}+q_{1}^{2}\right)}{8 \omega_{1}} \\
& -\frac{3 b_{1} q_{1}^{2}}{4 \omega_{1}}+\frac{3 b_{1}\left(p_{1} p_{2}+q_{1} q_{2}\right)}{4 \omega_{1}}+\frac{3 b_{1} q_{2} q_{1}}{2 \omega_{1}}, \\
& n_{21}=u_{2}-\frac{3 b_{2} p_{1} q_{1}}{4 \omega_{2}}+\frac{3 b_{2}\left(q_{1} p_{2}-p_{1} q_{2}\right)}{4 \omega_{2}} \\
& +\frac{3 b_{2} p_{1} q_{2}}{2 \omega_{2}}-\frac{3 b_{2} p_{2} q_{2}}{4 \omega_{2}} \\
& n_{22}=-u_{3}+\frac{3 b_{2} p_{1} q_{1}}{4 \omega_{2}}-\frac{3 b_{2} p_{2} q_{1}}{4 \omega_{2}}+\frac{3 b_{2} p_{2} q_{2}}{4 \omega_{2}}-\frac{3 b_{2} p_{1} q_{2}}{4 \omega_{2}}, \\
& n_{23}=-\frac{l_{2}}{2 \omega_{2}}-\frac{3 b_{2} q_{1}^{2}}{4 \omega_{2}}-\frac{3 b_{2}\left(p_{1}^{2}+q_{1}^{2}\right)}{8 \omega_{2}}-\frac{3 b_{2}\left(p_{2}^{2}+q_{2}^{2}\right)}{8 \omega_{2}} \\
& +\frac{3 b_{2} q_{1} q_{2}}{2 \omega_{2}}+\frac{3 b_{2}\left(p_{1} p_{2}+q_{1} q_{2}\right)}{4 \omega_{2}}-\frac{3 b_{2} q_{2}^{2}}{4 \omega_{2}} \\
& n_{24}=\frac{3 b_{2}\left(-p_{1}^{2}+q_{1}^{2}\right)}{8 \omega_{2}}-\frac{3 b_{2} q_{1} q_{2}}{2 \omega_{2}}-\sigma_{2}+\sigma_{1} \\
& +\frac{3 b_{2} q_{2}^{2}}{4 \omega_{2}}+\frac{3 b_{2}\left(p_{2}^{2}+q_{2}^{2}\right)}{8 \omega_{2}}+\frac{3 b_{2}\left(p_{1}^{2}+q_{1}^{2}\right)}{4 \omega_{2}} \\
& -\frac{3 b_{2}\left(p_{1} p_{2}+q_{1} q_{2}\right)}{4 \omega_{2}},
\end{aligned}
$$

$$
\begin{gathered}
n_{31}=\frac{3 b_{1} p_{2} p_{1}}{2 \omega_{1}}-\frac{3 b_{1}\left(p_{2}^{2}-q_{2}^{2}\right)}{8 \omega_{1}}+\sigma_{2}+\frac{3 b_{1}\left(p_{1} p_{2}+q_{1} q_{2}\right)}{4 \omega_{1}} \\
-\frac{3 b_{1}\left(p_{2}^{2}+q_{2}^{2}\right)}{4 \omega_{1}}-\frac{3 b_{1} p_{1}^{2}}{4 \omega_{1}}-\frac{3 b_{1}\left(p_{1}^{2}+q_{1}^{2}\right)}{8 \omega_{1}}, \\
n_{32}=\frac{l_{1}}{2 \omega_{1}}+\frac{3 b_{1} p_{2}^{2}}{4 \omega_{1}}+\frac{3 b_{1}\left(p_{2}^{2}+q_{2}^{2}\right)}{8 \omega_{1}}+\frac{3 b_{1}\left(p_{1}^{2}+q_{1}^{2}\right)}{8 \omega_{1}} \\
+\frac{3 b_{1} p_{1}^{2}}{4 \omega_{1}}-\frac{3 b_{1}\left(p_{1} p_{2}+q_{1} q_{2}\right)}{8 \omega_{1}}-\frac{3 b_{1} p_{2} p_{1}}{2 \omega_{1}},
\end{gathered}
$$$$
n_{33}=-u_{1}+\frac{3 b_{1} q_{1} p_{2}}{4 \omega_{1}}-\frac{3 b_{1} q_{2} p_{2}}{4 \omega_{1}}
$$$$
+\frac{3 b_{1} q_{2} p_{1}}{4 \omega_{1}}-\frac{3 b_{1} q_{1} p_{1}}{4 \omega_{1}}
$$$$
n_{34}=u_{1}+\frac{3 b_{1} q_{2} p_{2}}{4 \omega_{1}}-\frac{3 b_{1}\left(q_{1} p_{2}-p_{1} q_{2}\right)}{4 \omega_{1}}
$$$$
+\frac{3 b_{1} q_{1} p_{1}}{4 \omega_{1}}-\frac{3 b_{1} q_{2} p_{1}}{2 \omega_{1}}
$$$$
n_{41}=\frac{l_{2}}{2 \omega_{2}}+\frac{3 b_{2} p_{1}^{2}}{4 \omega_{2}}+\frac{3 b_{2}\left(p_{1}^{2}+q_{1}^{2}\right)}{8 \omega_{2}}+\frac{3 b_{2}\left(p_{2}^{2}+q_{2}^{2}\right)}{8 \omega_{2}}
$$$$
-\frac{3 b_{2}\left(p_{1} p_{2}+q_{1} q_{2}\right)}{4 \omega_{2}}-\frac{3 b_{2} p_{1} p_{2}}{2 \omega_{2}}+\frac{3 b_{2} p_{2}^{2}}{4 \omega_{2}},
$$$$
n_{42}=\frac{3 b_{2} p_{1} p_{2}}{2 \omega_{2}}+\sigma_{2}-\sigma_{1}-\frac{3 b_{2} p_{2}^{2}}{4 \omega_{2}}-\frac{3 b_{2}\left(p_{2}^{2}+q_{2}^{2}\right)}{8 \omega_{2}}
$$$$
-\frac{3 b_{2}\left(p_{1}^{2}+q_{1}^{2}\right)}{4 \omega_{2}}+\frac{3 b_{2}\left(p_{1} p_{2}+q_{1} q_{2}\right)}{4 \omega_{2}},
$$$$
n_{43}=u_{2}+\frac{3 b_{2} p_{1} q_{1}}{4 \omega_{2}}-\frac{3 b_{2}\left(p_{1}^{2}-3 q_{1}^{2}+2 p_{1} q_{2}\right)}{8 \omega_{2}}
$$$$
-\frac{3 b_{2} q_{1} p_{2}}{2 \omega_{2}}+\frac{3 b_{2} p_{2} q_{2}}{4 \omega_{2}}
$$$$
n_{44}=-u_{3}-\frac{3 b_{2} p_{1} q_{1}}{4 \omega_{2}}+\frac{3 b_{2} p_{1} q_{2}}{4 \omega_{2}}-\frac{3 b_{2} p_{2} q_{2}}{4 \omega_{2}}+\frac{3 b_{2} p_{2} q_{1}}{4 \omega_{2}} .
$$

\section{Acknowledgment}

The authors thank Dr. Weihua Su who helped them during the writing of this paper.

\section{References}

[1] C. L. Lee and N. C. Perkins, "Nonlinear oscillations of suspended cables containing a two-to-one internal resonance," Nonlinear Dynamics, vol. 3, no. 6, pp. 465-490, 1992. 
[2] J. Li, X. Guo, K. Yang, and Y. Yan, "Study on the nonlinear vibration of axially moving cylindrical shells made from composites," Chinese Journal of Solid Mechanics, vol. 32, no. 2, pp. 176-185, 2011.

[3] Z. Xin, S. Dagang, S. Yang et al., "Analysis of damping vibration reduction performance of viscoelastic shocker absorber under low frequency and heavy loading," China Mechanical Engineering, vol. 23, no. 14, pp. 1651-1656, 2012.

[4] L. Xinye, Z. Lijuan, and Z. Huabiao, "Forced vibration of a gyroscope system and its delayed feedback control," Journal of Vibration and Shock, vol. 31, no. 9, pp. 63-68, 2012.

[5] T. Inoue and Y. Ishida, "Nonlinear forced oscillation in a magnetically levitated system: the effect of the time delay of the electromagnetic force," Nonlinear Dynamics, vol. 52, no. 1-2, pp. 103-113, 2008.

[6] B. Y. Moon and B. S. Kang, "Dynamic analysis of harmonically excited non-linear system using multiple scales method," KSME International Journal, vol. 16, no. 6, pp. 819-828, 2002.

[7] P. Xian and Z. Shixiang, "Nonlinear resonance response analysis of a kind of passive isolation system with quasi-zero stiffness," Journal of Human University (Natural Sciences), vol. 38, no. 8, pp. 34-39, 2011.

[8] Y. Wang, B. Zheng, and C. Zhang, "An algebraic criterion of zero solutions of some dynamic systems," Abstract and Applied Analysis, vol. 2012, Article ID 956359, 13 pages, 2012.

[9] S. Chen, X. Xinxi, G. Zhenhai et al., "Analysis on twolevel damping efficiency and recumbent comfort for tracked emergency ambulance," Journal of Vibration, Measurement \& Diagnosis, vol. 32, no. 5, pp. 754-857, 2012.

[10] L. Shuang, L. Yangshu, L. Bin et al., "Parametric vibration analysis and control in coupling rotating mechanical drive system," China Mechanical Engineering, vol. 23, no. 12, pp. 14611466, 2012.

[11] L. Haoran, Z. Zhanlong, S. Peiming et al., "Stability control of a coupled nonlinear torsional vibration system," Journal of Vibration and Shock, vol. 30, no. 9, pp. 140-144, 2011.

[12] G. Zhu and J. Wei, "Stability and hopf bifurcation analysis of coupled optoeletronic feedback loops," Abstract and Applied Analysis, vol. 2013, Article ID 918943, 11 pages, 2013.

[13] R. W. Ibrahim, "Stability and stabilizing of fractional complex lorenz system," Abstract and Applied Science, vol. 2013, Article ID 127103, 13 pages, 2013.

[14] J. Ma, B. Zheng, and C. Zhang, "A matrix method for determining eigenvalues and stability of singular neutral delaydifferential systems," Abstract and Applied Analysis, vol. 2012, Article ID 749847, 11 pages, 2012. 


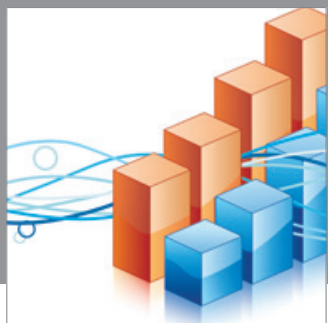

Advances in

Operations Research

mansans

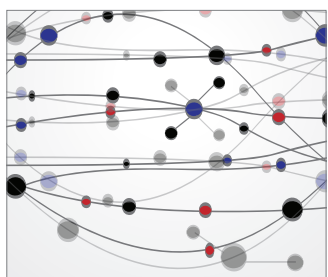

The Scientific World Journal
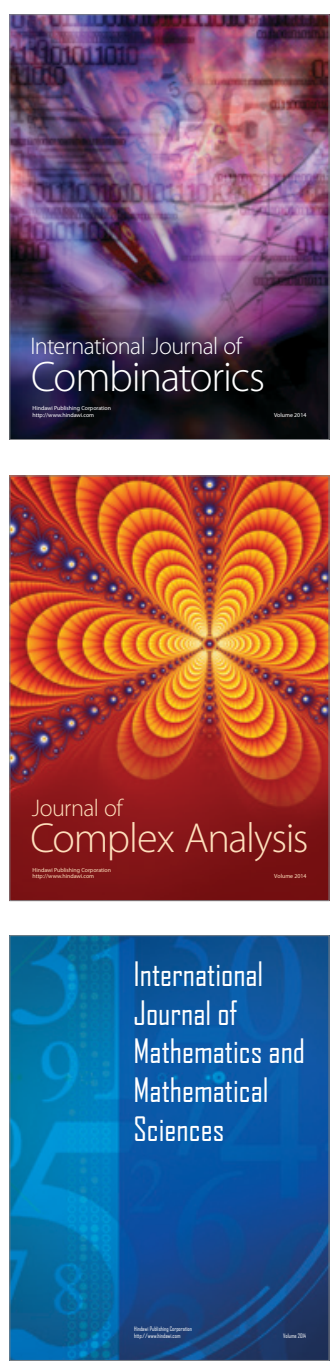
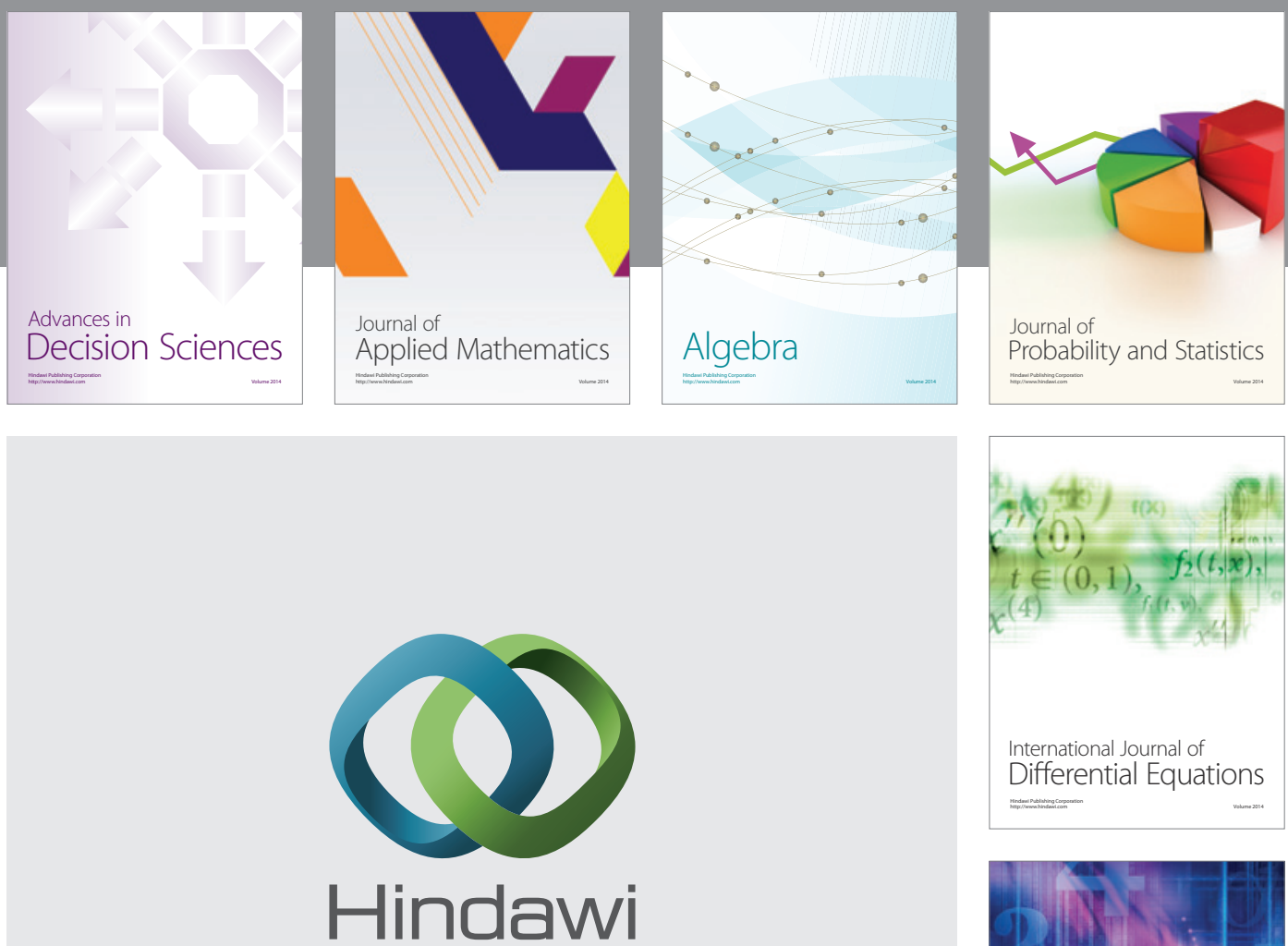

Submit your manuscripts at http://www.hindawi.com
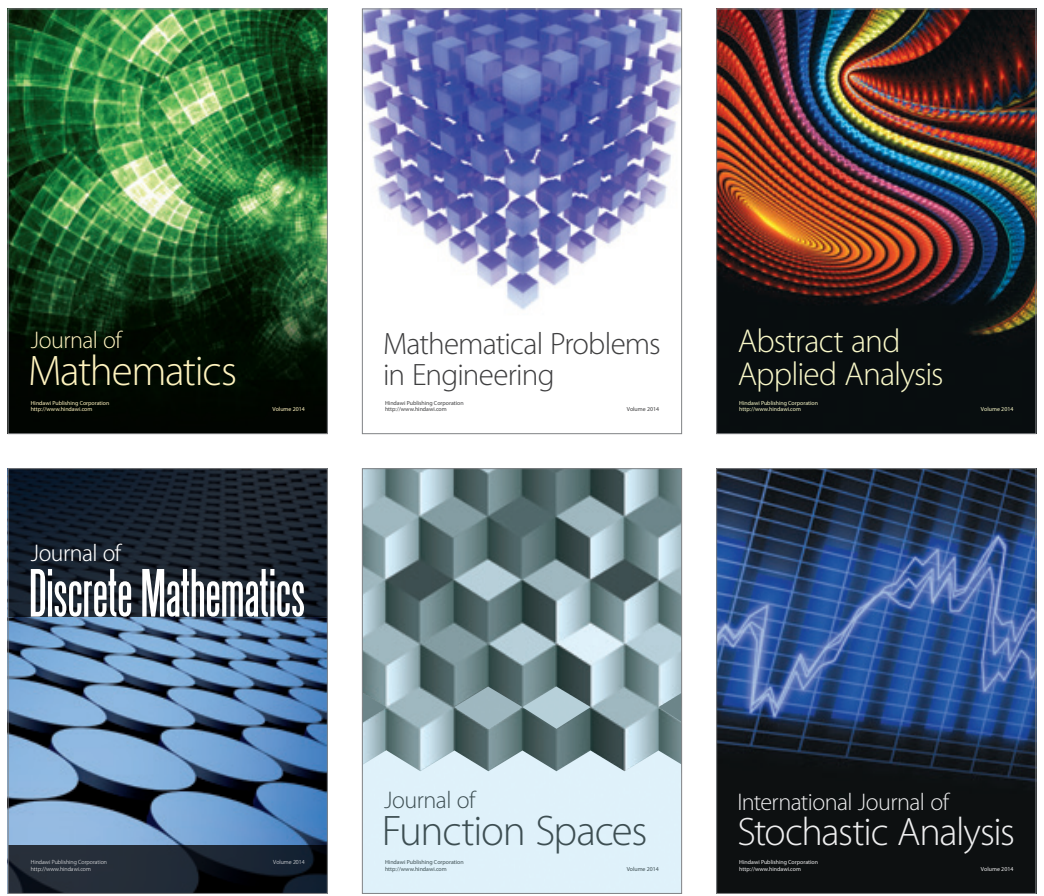

Journal of

Function Spaces

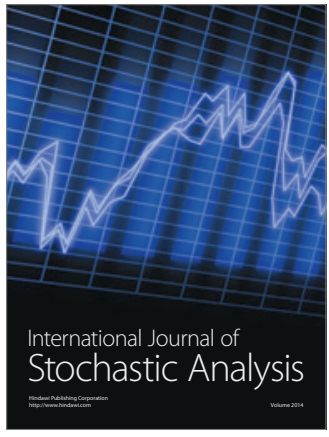

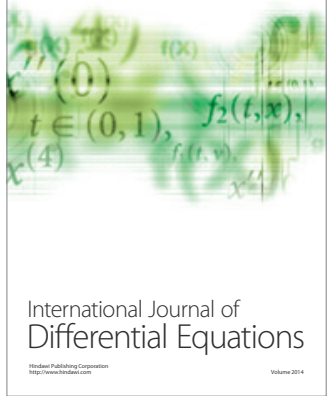
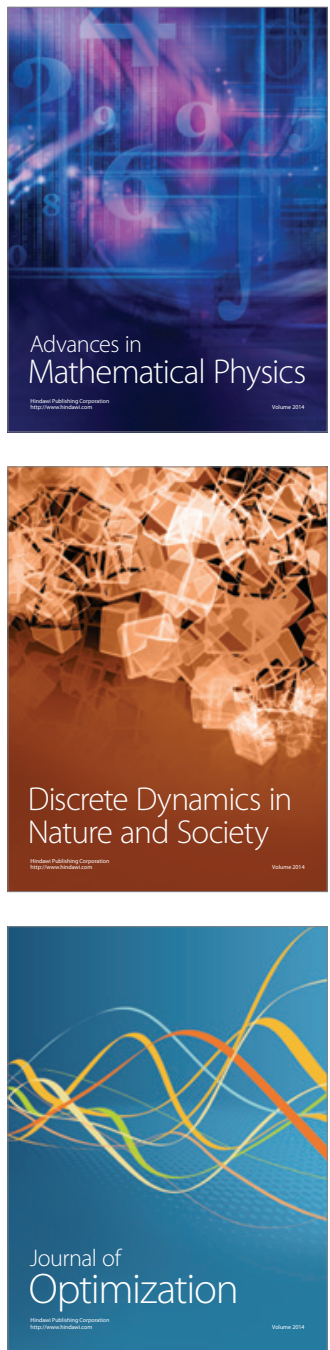